\title{
Oralidad y escritura en la lirica griega arcaica: el ejemplo de Píndaro
}

\author{
DANIEL A. TORRES \\ Universidad Nacional del Sur \\ Universidad Nacional de La Plata \\ República Argentina
}

\begin{abstract}
RESUMO: Neste artigo focaliza-se o estudo da poesia de Píndaro, a partir das recentes abordagens hermenêuticas que consideram a cultura oral da Grécia arcaica como um fator decisivo para a interpretação da poesia arcaica. O crítico status quaestionis é revisado, especialmente em relação ao problema da elocução em primeira pessoa nas epinícias, como também a sua atribuição ao coro ou ao poeta, o que leva à recente discussão sobre a intervenção regular dos coros na performance. A hipótese de uma performance solo parece estar relacionada com um grau de conhecimento e o uso da escrita no âmbito da composição. Algumas marcas textuais nas odes de Pindaro que indicam escrita são analisadas e confrontadas com sua estrutura. Este estudo procura dar uma contribuição à recente questão do caráter coral das epinícias.
\end{abstract}

PALAVRAS-CHAVE: Poesia de Píndaro; epinícias; coro; performance.

La tendencia generalizada en la crítica pindárica de los últimos años ha sido contextualizar la producción poética de Píndaro en el marco de la cultura oral de la Grecia arcaica, cuestión clave para la comprensión moderna de Píndaro, inevitablemente leído como texto, y al que se suma el peso de la tradición del comentario textual en la multiplicadora extensión de los escolios. Pareciera que, para aproximarse al contexto oral de la ejecución de una oda pindárica, buena parte de la tarea hermenéutica que tenemos por delante habría de consistir en desmontar todo este bagaje, en lo que parece un intento por "des-escribir" lo ostensiblemente escrito. Basadas sobre lo escrito, ni la teoría literaria moderna ni la antigua, con sus minuciosas clasificaciones, resultan herramientas suficientes a la hora de comprender una oda pindárica. Esto es, ciertamente, consecuencia del descrédito progresivo de los comentaristas antiguos, variadamente acusados de parcialidad, de falta de perspectiva y hasta de simple y pura invención (cfr. Wilson, 1980; Lefkowitz, 1980 y 1981, p. 57-66; Slater, 1971 y 1977). De ahí la necesidad de recurrir a enfoques de otras disciplinas, especialmente de la antropología (cfr. especialmente Nagy, 1990, p. 1-16), que han llevado a plantear la discusión acerca de la oralidad y la escritura en la Grecia arcaica más allá de lo estrictamente literario. Pero lo que interesa ver aquí es, por un lado, cómo estos problemas ya venían configurándose en la crítica pindárica especializada de los últimos años y, en segundo lugar, atender brevemente a un testimonio de Píndaro que impone cierta cautela a las generalizaciones sobre la oralidad de la lírica. 
La crítica pindárica moderna, caracterizada por un agonístico disenso (cfr. Young, 1970 , p. 1-95, esp. p. 91; cfr. también Heath, 1986), ha dado un nuevo giro a las lecturas anteriores de la poesía de Píndaro desde los estudios de Elroy Bundy (1962), mediante la reducción de los diversos elementos detectados en los epinicios al único y exclusivo objetivo del elogio. Bundy explicó la diversidad de elementos que el poeta va integrando en una composición dada como múltiples contrastes ("foils"), sin significación propia más allá de la funcional, para mejor realzar la figura del laudandus. Con esto echaba por tierra juntamente las alusiones al contexto histórico y la importancia de los enunciados en primera persona, fundando una nueva aproximación al texto diametralmente opuesta a las dos tendencias entonces dominantes en la crítica: por un lado, al historicismo, propio del acercamiento más erudito y, por otro lado, a una estética con una doble vertiente: una romántica, que explicaba las rupturas y abruptas transiciones típicas de Píndaro recurriendo a la idea de la inspiración irracional que guiaba al poeta, y la vertiente más moderna, derivada de la "Nueva Crítica", que intentaba la explicación de los poemas individuales como mónadas autosuficientes, oponiéndose a los enfoques historicistas y particularmente a los biográficos.

El problema de la oralidad constituye en este panorama crítico una cuestión de fondo, y esto se debe en buena medida al hecho de que Bundy termina transponiendo al estudio de los epinicios el bagaje formulario detectado en la épica, sobre todo desde los estudios de Milman Parry. Así Bundy plantea el estudio retórico de los poemas, señalando el carácter convencional de muchos elementos que hasta entonces se habían tomado aisladamente en el contexto de cada poema, y que ahora aparecían como topoi, equivalentes a las fórmulas de la épica.

Como es sabido, el punto de partida para la teoría de la oralidad está dado por las investigacioens de Milman Parry y su seguidor Lord (Parry, 1928-1971; Lord, 1960; cfr. las discusiones en Gentili, 1984, p. 6-30; Thomas, 1992, esp. p. 29-51; Ong, 1987/82, p. 25-37; Holoka, 1991, esp. p. 457-466). Según esta perpectiva, se desdibuja progresivamente la figura del poeta para dar paso a la importancia de la audiencia y de la ejecución (performance), conduciendo a establecer una diferencia radical entre poesía oral y poesía escrita, que se ha vuelto hoy cuestionable. En efecto, en un libro reciente, con nuevos enfoques en el estudio de la oralidad y la escritura en la Grecia arcaica, Rosalind Thomas (1992) examina la interpenetración entre oralidad y escritura, de lo que resulta la constatación de una gran variabilidad y complejidad en las culturas orales, contra la tesis que ve al poeta y la poesía oral como herramientas mecánicas de la tradición. Por otro lado, las diferencias de calidad en la producción poética oral de distintas culturas evidencia que la teoría de Parry y Lord, que termina negando valor artístico a la poesía oral, no puede aceptarse como concluyente con respecto a Homero. De ahí la reacción estética que vuelve al estudio de las cualidades literarias de Homero, restableciéndose la figura del poeta. Thomas propone reconsiderar el papel de la memorización y de la reflexión privada del poeta, lo que conduce a aceptar la elaboración compositiva para la poesía oral, contra la tesis de la improvisación oral formularia en la línea Lord-Parry; así, el carácter formulario no aparece ni como necesario ni como exclusivo para la poesía oral. Esta perpectiva implica, en última instancia, restituir al poeta su cualidad creadora. Transferido a la lírica, implica reconsiderar el valor de los enunciados en primera persona, y en la crítica pindárica específicamente, después de la desaparición del "yo poético" de un anacrónico romántico tras un profesional que produce discursos de alabanza pública atento a las convenciones de su época, vuelve a emerger la "mente maestra" 
(así Lloyd-Jones, 1990) que organiza todo el material tradicional en base al conocimiento de una techne.

Para la rehabilitación de la primera persona poética en los epinicios Mary Lefkowitz (1980 y 1981) ha hecho su contribución importante al considerar las autorreferencias como elemento ficticio; esto es, deben ser leídas en relación al "yo poético" que compone y no al que ejecuta, es decir, al poeta y no al coro. Siguiendo la línea de Bundy en cuanto al rechazo de la alusión histórica en los enunciados en primera persona, el concepto superador con respecto a la teoría de Bundy radica en restablecer la figura del poeta en el acto de composición, diluyendo por otro lado la importancia del coro en el acto de ejecución o performan$c e$. Esto implica, en última instancia, una modificación profunda que atañe a nuestra comprensión de la ocasión y su contexto, como lo muestran algunos estudios críticos recientes ${ }^{\perp}$, que llegan a poner en tela de juicio la clasificación misma de la lírica arcaica.

En cuanto a la oralidad de la performance, uno de los puntos cuestionados es el de la existencia de una performance regular en todos los casos. Varias odas de Píndaro parecen haber sido compuestas directamente para el destinatario, para ser leídas en público o en privado, en círculos más o menos restringidos según los casos. Pero si queremos atender a la ocasión de la performance, hoy no podemos dejar de reconocer su pertenencia al contexto religioso, que determina el sentido primario de la ocasión. De ahí la insuficiencia de categorías como la de "género" aplicada al epinicio como forma compositiva específica: no hay allí nada que pueda parecerse a lo que modernamente se ha entendido -sin consenso alguno - por el término "género"2; y esta es una de las consecuencias más importantes en el cuestionamiento de Davies a la clasificación oficial, institucionalizada en nuestras universidades, de la lírica griega arcaica.

Davies (1988) cuestiona la división tradicional entre monodia y lírica coral, completamente arraigada en el estudio y la enseñanza de la literatura griega. Las argumentaciones en contra de esta clasificación se basan en los nuevos resultados arrojados por la investigación, por un lado, sobre los líricos arcaicos, especialmente Estesícoro ${ }^{3}$, y por otro lado, sobre la significación de la primera persona del singular en los llamados líricos corales, especialmente Píndaro, a partir de los estudios de Mary Lefkowitz (1980). Como evidencia interna tomada del corpus pindárico mismo, se consigna el hecho de que sus encomios eróticos (Frag. 123 y 124ab Sn.) no presentan indicios de la presencia de un coro $^{4}$, y otro tanto ocurre con Baquílides ${ }^{5}$ En los epinicios mismos pueden detectarse ciertos rasgos, indicios o autorreferencias: la Pítica 4, con su extensión de casi trescientos versos en estilo épico, a la manera de Estesícoro, resulta más verosímil si se la entiende como monodia. Wilamowitz (1922) había postulado el carácter monódico para las Olímpicas 1 y 2. Mary Lefkowitz (1985) observó que fuera de los escolios a Píndaro no hay evidencia de que todas las odas fueran corales, y Aristófanes Nubes 1355ss. se toma como prueba de que para un espectador del siglo V no era incongruente la ejecución monódica de un epinicio de Simónides (Frag. 507 P) $)^{6}$.

En cuanto a los testimonios antiguos sobre el tema, Davies cita dos pasajes de las Leyes de Platón (764d-e y 700ab) como prueba de la incomprensión moderna que ha generado la falsa clasificación dicotómica entre lírica monódica y coral. El primero de estos pasajes ha sido tomado como prueba de que Platón conocía una división de la mousike entre monodia y canto coral, pero el segundo pasaje, una breve reseña histórica de la poesía, no presenta esa distinción. Así la división moderna no tiene punto de apoyo en las teorías antiguas, ni en las del Renacimiento. La distinción entre monodia y canto coral aparece 
recién en los escritos de los hermanos Schlegel (1802), y es K. O. Müller (Londres, 1858) quien lo introduce en el campo de la filología clásica, en su historia de la literatura griega, que había de ser decisiva en el siglo XX (hasta Lesky inclusive). Davies concluye que, si bien puede ser lícito hablar de poesía monódica y de poesía coral, no puede hacerse extensiva esta distinción a los poetas mismos, que contrariamente parecen haber practicado ambos modos de composición.

En el mismo sentido, Mary Lefkowitz (1988) plantea la diferenciación genérica entre lírica monódica y lírica coral como postulado del siglo XIX (Müller 1841, Bergk 1883, Wilamowitz 1889, Reisch 1899, etc.) sin fundamento en las autoridades antiguas, que clasificaban los distintos tipos de poesía según la función (i.e., destinatario y ocasión). M. Lefkowitz considera que ante la ausencia de distinción entre monodia y coral en las fuentes antiguas, es lícito suponer una combinación de ambos modos de representación?.

Sobre la hipótesis de que los poemas de Estesícoro no eran cantados por un coro, la estructura triádica ya no denota necesariamente una representación coral ${ }^{8}$. M. Lefkowitz plantea entonces el reexamen de algunos pasajes pindáricos, tradicionalmente interpretados sobre el presupuesto del canto coral, partiendo del presupuesto contrario de que la oda era cantada como un solo, con o sin acompañamiento de danza coral ${ }^{9}$.

De este examen se desprende el cuestionamiento a los presupuestos antiguos y modernos sobre la representación coral. Puede pensarse entonces en una composición monódica para la Pítica 4, y sugerir incluso que todos los epinicios fueran esencialmente monódicos, con o sin acompañamiento de danza. Las pinturas de vasos añaden un testimonio a favor de la representación monódica de las celebraciones de victorias. Esta hipótesis incide en el problema del "yo poético", que dejaría de referirse a la voz convencional del coro, para representar la autoridad del poeta ante el contrato público del epinicio. Pero, por otro lado, el alcance de la función pública se ve en cierto modo reducido al alcance de publicidad de algunos tipos de monodia.

En la misma línea de M. Lefkowitz, Heath (1988) examina el término kômos y sus derivados, atendiendo especialmente a su vinculación con el contexto simposíaco. También Heath cuestiona el rol del coro, su grado de entrenamiento y su función precisa, esto es, si el coro canta o baila o ambas cosas. Importa destacar que los testimonios que Heath va aduciendo contribuyen a desdibujar el carácter ya casi fantasmagórico de los coros líricos, pero el argumento más significativo es que, si estos coros deben tomarse como un elemento programático esencial, entonces Píndaro se ha limitado a unas referencias demasiado escasas. Y estamos en el mismo punto que M. Lefkowitz y Davies: hay que releer el texto de Píndaro cambiando los presupuestos.

Como parte del contexto oral, debe tenerse en cuenta el aspecto ritual que acompaña a los epinicios. Las celebraciones de victoria incluyen sacrificios y festejos, para lo cual se organizan los kômoi, con danza improvisada. Los cantos podrán tener lugar en el camino, a la llegada y en el simposio después de la fiesta. Se ha postulado una distinción en los cantos de victoria: unos simples, familiares, fácilmente representados de manera improvisada por el kômos, y luego las odas más complejas, encargadas, como las de Píndaro y Baquílides, representadas por un solista, el poeta o un delegado: "most of the commissioned odes that we possess were probably written to be performed solo on arrival " (Heath, 1988, p. 193).

Pese a que esta discusión todavía presenta un carácter conjetural, su mérito es poner en evidencia ese mismo carácter conjetural de muchos postulados acerca de la literatura griega. 
Hasta aquí hemos visto algunos problemas de crítica pindárica que, en medio del auge de la oralidad y sus diversas manifestaciones, desembocan en una hipótesis que, llevada a sus últimas consecuencias, termina por presentar una situación del poeta que se acomoda más a la composición escrita que a la oral. En efecto, entre los líricos arcaicos Píndaro es el poeta que más cercano se muestra a los efectos atribuidos a la progresiva difusión de la escritura. Uno de los rasgos inmediatamente observables, el de la oscuridad de expresión con la concomitante dificultad de interpretación, tiene que ver precisamente con esto. Hoy en día se ha trabajado bastante para demostrar que esa oscuridad es producto de la comprensión moderna de Píndaro, y que no hay fuentes antiguas que la atestigüen fuera de los escolios $^{10}$. Pero por otro lado también se considera, junto a la performance, la instancia, o mejor dicho las instancias de re-performance, por la dificultad de lenguaje y por la función programática misma propia del epinicio de difundir los méritos del vencedor (así Ruth Scodel, 1996, p. 60-61). De hecho, la comprensión moderna viene condicionada por Eustacio, en cuyo Prooemium se da por presupuesto el uso de la escritura por parte de Píndaro, al menos en la instancia de la composición, al punto de explicar determinados rasgos sintácticos y retóricos en función del uso de la escritura. Así, al explicar el uso de la digresión en Píndaro (parékbasis), señala (Eustathii prooemium 4, Drachmann III, p. 287)"1:

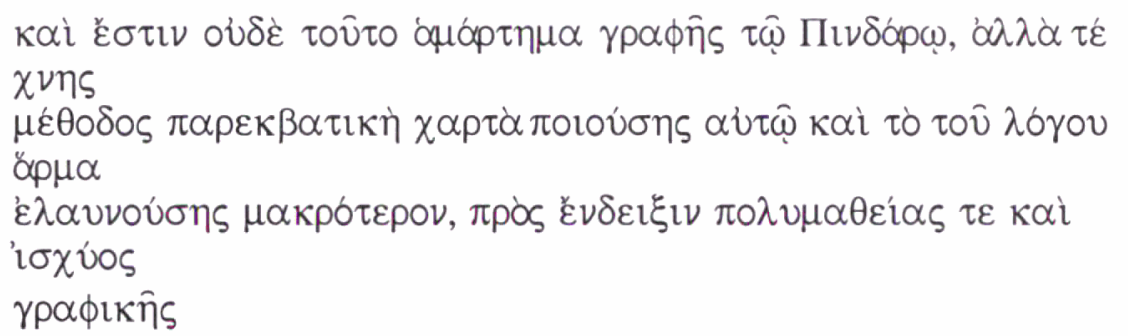

Y es que, más incluso que la Musa arrebatadora o el supuesto genio irracional del poeta, el uso de la escritura en la instancia de composición resulta una de las mejores y simples herramientas para explicar las abruptas transiciones y las digresiones. Que en Píndaro la escritura presente un grado de internalización significativo se prueba por el texto mismo. Uno de los pasajes frecuentemente citados como testimonios de los primeros usos de la escritura es el proemio de la Olímpica 10, que presenta precisamente ese grado de internalización:

Tòv 'O

'A

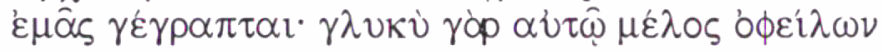
$\dot{\varepsilon} \pi \imath \lambda \dot{\varepsilon} \lambda \alpha \theta^{\circ}$.

"Leedme al vencedor olímpico hijo de Arquestrato, en qué parte de mi mente está inscripto, pues he olvidado que le debo el dulce canto".

Si leemos este pasaje sobre el presupuesto de la ejecución coral, ¿qué implicaría el uso de la primera persona como voz colectiva? La primera y más evidente respuesta apunta a una amplia difusión de la escritura, al punto de que el coro, representante de la comunidad cívica, llegue a manifestar ese grado de compenetración con ella. Y aunque recientemente se ha señalado el elevado porcentaje de sofisticación que presentan las lecturas eruditas de 
la lírica arcaica (Scodel, 1996, p. 68-69), es en verdad demasiado sofisticado no ver en el proemio de $\mathrm{Ol} .10$ un anuncio manifiesto de la primera persona poética, en el acto mismo de

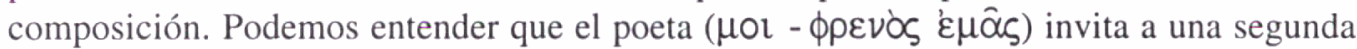
persona ( $(\alpha \nu \alpha \gamma \nu \omega \tau \varepsilon$ ), identificada en los versos siguientes como la Musa y Alatheia, a leer, a proferir en voz alta, como el heraldo en el anuncio público, el nombre del vencedor ${ }^{12}$. Hay lo que podríamos llamar un reflejo de la performance, en tanto el poeta busca recrear para la audiencia el acto primero de la proclama pública, pero ese reflejo se da en una instancia de intermediación, esto es, se lee lo ya inscripto, y el punto de partida de la oda es la inscrip-

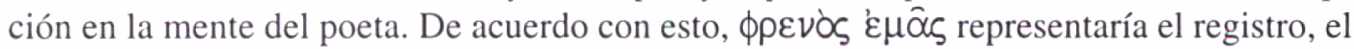
archivo poético, que el poeta busca asimilar a los registros de los Juegos. En ese acto primario que consagra al vencedor, haciéndolo adicionalmente merecedor del elogio público, el poeta escoge una expresión que pone en evidencia el rol decisivo de la escritura. Lo que se da por los medios orales es precisamente la parte de la publicidad, sea el anuncio del heraldo o la performance del epinicio, mientras que lo escrito implica cierto grado de privacidad y de reserva, que incluso hace que, a la manera platónica en el Fedro (274e-275b), pueda ser pasado por alto y olvidado ( $\varepsilon \pi i \lambda \dot{\varepsilon} \lambda \alpha \theta^{\circ}$ ), como lo consigna aquí el poeta. La expresión de auto-confianza en la perdurabilidad de su canto, un topos especialmente apto para la autoafirmación de poemas orales, aparece ahora como un indicio a favor de la conciencia poética, por parte de Píndaro, de que su transmisión se apoyaba en la escritura ${ }^{13}$.

Finalmente queremos apuntar una reflexión: la crítica más reciente ha ido explicando la dificultad u oscuridad de Píndaro por el distanciamiento que supone entre el poema y un lector moderno todo el bagaje que comporta la performance. Si tomamos el eventual texto escrito como simple ayuda-memoria que el mismo poeta o el director del coro habrían usado como base para la ejecución pública de la oda, todos los componentes adicionales al texto se pierden para la experiencia del lector moderno, y el remedio de una buena dosis de imaginación para reconstruirlo (así Thomas, 1992, p. 117) se acomoda poco a los criterios que hemos convenido en llamar científicos. Ante este estado de cosas, ¿no es más honesto y sencillo tratar de recuperar el significado del texto reducido a su condición de "ayudamemoria"? Todo lo perdido de la irrecuperable performance es análogo a los significados condensados en el texto, y si se nos propone la imaginación como herramienta adecuada para recuperar algo de la performance, entonces quizás hagamos mejor empleándola para la indagación de nuevos significados, o para una nueva combinación de los significados de siempre.

\section{Notas}

1 - Davies (1988); Mary Lefkowitz (1988); Heath (1988); cabe destacar que estamos lejos de una discusión consensuada; véanse las objeciones planteadas por Anne Burnett (1989) a estos estudios. Ruth Scodel (1996) si bien no se ocupa directamente de este problema, muestra la actualidad del mismo; también Miller (1993).

2 - Para el concepto de género como resultado de un proceso evolutivo a partir de la ocasión ritual originaria, véase Nagy (1990) p. 116ss.

3 - Los argumentos de Davies concernientes a los poetas líricos pueden resumirse en los siguientes puntos: 1.- Estesícoro: su estilo épico, así como la longitud de sus narraciones, hacen inverosímil que se trate de un poeta coral. Su asociación con Homero desde 
la antigüedad apuntan igualmente en el sentido de la monodia. La división triádica de sus estrofas ha sido entendida como un principio de composición puramente musical, sin evidencia de su carácter coral (West 1971). 2.- En cuanto a Ibico: ninguna autoridad antigua señala que sea coral, opinión que se ha formado por su frecuente asociación con Estesícoro. Los fragmentos 286 y $287 \mathrm{P}$ han sido tomados como ejemplos de monodia lírica por varios filólogos (Lattimore 1960, Campbell 1967, Kirkwood 1974), aunque no hicieron extensiva esta nueva clasificación al resto de sus poemas. 3.- Alcman: su fama se fundamenta en los partheneia desde la antigüedad, pero no hay evidencia de que fuera el único tipo de composición que practicó. La introducción de la primera

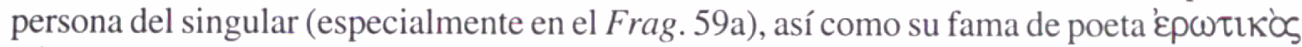
$\pi$ róv (Suda s.v.) apuntan a la monodia.

4 - Van Groningen (1960) ya había observado que en estos tipos de poemas no hay huellas de ejecución coral, sino que presentan los rasgos propios de una monodia.

5 - (Frag. 20B 1ss.; cfr. 20C 1ss.). Como el lenguaje de estos fragmentos recuerda a Píndaro Ol. $1.17 \mathrm{ss}$., la argumentación contra el carácter coral se extiende a los epinicios.

6 - La lista puede ampliarse fácilmente con Píndaro: Pít. 3 e Ist. 2, las llamadas epístolas, Ol. 6 con la figura del intermediario Eneas (vv. 87ss.). El argumento contra las

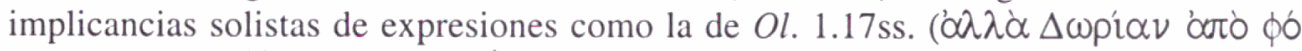

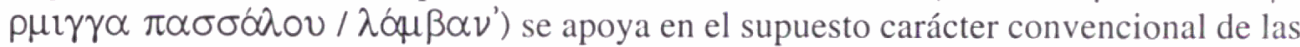
mismas, sin que deba colegirse de ello que fueran efectivamente monodias. Davies propone el procedimiento contrario: dada la vaguedad en las referencias pindáricas a los coros-cantantes, bien puede entenderse que estas últimas sean convencionales.

7 - Como se advierte en Homero: Il. 9.186-9 monodia; Il. 22.391-2 coro (cfr. Il. 1.472-4); Od. 8.261-4 combinación de un solo con un coro que danza; cfr. $I l$. 18.569-72; Od. 23.143-7). M. Lefkowitz aporta testimonios que indican la combinación de un cantante solista con un coro que acompaña danzando: i) Estesícoro, cuyo nombre indica la introducción de la danza, no del canto coral, ya que las fuentes antiguas lo presentan como un solista a la manera del rapsoda homérico; ii) Pínd. Pít. 1.1ss. e Himno Hom. Apolo 182-206, donde los cantantes no danzan y los bailarines no cantan.

8 - Los escolios métricos a Píndaro vinculan los términos estrofa, antiestrofa y epodo a los movimientos del coro, pero no hay fuentes anteriores a los bizantinos; en todo caso, la explicación concierne a la danza y no al canto (cfr. Drachmann III, p. 306, 311).

9 - Dichos pasajes son: Ol. 1.17-8; Pít. 10.55-9; Ist. 8.3-4; Pít. 5.22-3, 98-100; Pít. 3.73; y especialmente $\mathrm{Ol}$. 6.86-92 y Nem. 3.3-12. En estos últimos dos casos se examina la información de los escolios, que no pueden tomarse como autoridad en este punto dada la variedad de posibilidades que ofrecen. En todo caso, el texto de Píndaro acepta la posibilidad de que estos pasajes se refieran a un solista, y los coros presentes serían bailarines (Ol. 6) o el sonido de la conversación en un banquete (Nem. 3; cfr. Pít. 1.97-8 con escolio).

10 - Cfr. esp. Most (1985) p. 11-41; también Fitzgerald (1980) p. ix-x. Por su parte Scodel (1996; p. 60) sostiene que la dificultad de lenguaje es indicio de que se esperaba que individuos de la audiencia escucharan el poema varias veces.

11 - "Y tampoco esto es defecto de la escritura para Píndaro, sino un método digresivo del arte, que produce cosas agradables para él y que impulsan más lejos el carro de la palabra, para demostrar su ciencia múltiple y la fuerza de lo escrito". 


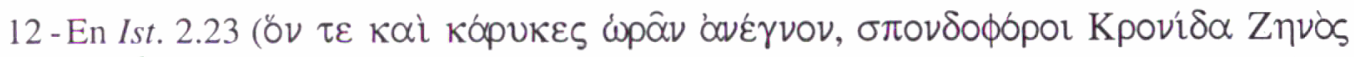
$\mathrm{A} \lambda \varepsilon \hat{\imath} \mathrm{ol}$ ) hay asimilación entre la proclama del vencedor y el acto de lectura, lo que lleva a la ecuación entre el acto meritorio para esa lectura, i. e. la victoria en un certamen de los Juegos, y el proceso de inscripción de tal hecho en el fluir continuo de la realidad. 13 - En este sentido es significativa la reiterada mención del tiempo en Ol. 10.7-8 y 53-55.

\section{Referências bibliográficas}

BUNDY, E. L. Studia Pindarica I \& II. Berkeley and Los Angeles: University of California Press, 1962.

BURNETT, Anne. Performing Pindar's odes. CP 84, p. 283-293, 1989.

DAVIES, M. Monody, choral lyric, and the tyranny of the handbook. CQ 38, p. 52-64, 1988.

DRACHMANN, A. B. Scholia vetera in Pindari carmina. Lipsiae: Teubner I 1903, II 1910, III 1927.

FITZGERALD, William. Agonistic poetry. Berkeley and Los Angeles: Univ.of California Press, 1987.

GENTILI, Bruno. Poesia e pubblico nella Grecia Antica. Bari: Laterza, 1984.

HEATH, Malcolm. The Origins of modern Pindaric criticism. JHS 106, p. 85-98, 1986.

. Receiving the kômos: the context and performance of epinician. AJP 109, p. 180$195,1988$.

HOLOKA, J. P. Homer, oral poetry theory, and comparative literature: Major Trends and Controversies in 20th Century Criticism. In: Latacz, J. (Ed.). C. R. 2. p. 456-481.

LATACZ, J. (Ed.) Zweihundert Jahre Homer-Forschung. Colloquium Rauricum Band 2. Stuttgart und Leipzig: Teubner, 1991.

LEFKOWITZ, Mary R. The influential fictions in the scholia to Pindar's Pythian 8. Classical Philology 70; p. 173-185, 1975.

. The victory ode. An Introduction. N.J.: Park Ridge, 1976.

. Autobiographical fiction in Pindar. HSCP 84, p. 29-49, 1980.

. The lives of the Greek poets. Baltimore: The Johns Hopkins University Press, 1981.

. Who sang Pindar's Victory Odes? AJP 109, p. 1-11, 1988.

LLOYD-JONES, H. "Pindar". In: Greek epic, lyric and tragedy. The academic papers of Sir Hugh LLoyd-Jones. Oxford: Clarendon Press, 1990; p. 57-79.

LORD, A. B. The singer of tales. Cambridge-Mass, 1960.

MILLER, A. M. Pindaric mimesis: The associative mode. CJ 89, p. 21-53, 1993.

MOST, Glenn. The measures of praise. Structure and function in Pindar's second Pythian and seventh Nemean Odes. Göttingen: Hypomnemata 83, Vandenheck-Ruprecht, 1985. 
NAGY, G. Pindar's Homer. The lyric possession of an epic past. Baltimore and London: The Johns Hopkins University Press, 1990.

ONG, W. J. Orality and literacy. The technologizing of thewWord. London: Methuen, 1982. (Versión en español, México, FCE, 1987).

PARRY, Milman. The making of the homeric verse. The Collected Papers of Milman Parry. Oxford: Ed. Adam Parry, 1971.

SCODEL, Ruth. "Self-correction, spontaneity, and orality in archaic poetry". In: Worthington, Ian (Ed.); p. 59-79.

SLATER, W. J. Pindar's house. GRBS 12, p. 141-152, 1971.

. Doubts about pindaric interpretation. Cj 72, p. 193-208, 1977.

THOMAS, Rosalind. Literacy and orality in ancient Greece. Cambridge: Cambridge University Press, 1992.

WEST, M. L. Stesichorus. CQ 21, p. 302-314, 1971.

WILSON, Penelope. "Pindar and his reputation in Antiquity". Proceedings of the Cambridge Philological Society 26, 1980, p. 97-114.

WORTHINGTON, Ian (Ed.) Voice into text. orality \& literacy in ancient Greece. Leiden: Brill, 1996.

YOUNG, David C. "Pindaric Criticism”. In: CALDER, W. M. \& STERN, J. (Ed.). Pindaros und Bakchylides. Wege der Forschung. Darmstadt, 1970; p. 1-95.

TORRES, Daniel. Orality and literacy in Greek archaic lyric poetry: the case of Pindar. Classica, São Paulo, 11/12, 195-203, 1998/1999.

SUMMARY: This communication focuses the study of Pindar's poetry starting from the recent hermeneutic approaches that view the oral culture of archaic Greece as a decisive factor for the interpretation of archaic poetry. The critical status quaestionis is revised, specially in relation to the problem of the utterances of the first person in the epinicians, so as of their attribution to the chorus or to the poet, which lead to the recent discussion about a regular intervention of choruses in the performance. The hypothesis of a solo performance appears to be related to a significant degree of knowledge and use of writing in the instance of composition. Some textual marks in Pindar's odes that indicate writing are analyzed and confronted with this critical framework. This study means a contribution to the recent question of the choral character of the epinicians.

KEY-WORDS: Pindar's poetry; epinicians; chorus; performance. 